\title{
Pancreatic Intraductal Papillary Mucinous Neoplasm, Pancreatobiliary-Type
}

National Cancer Institute

\section{Source}

National Cancer Institute. Pancreatic Intraductal Papillary Mucinous Neoplasm,

Pancreatobiliary-Type. NCI Thesaurus. Code C95512.

A pancreatic intraductal papillary mucinous neoplasm characterized by the presence of neoplastic epithelial cells that form thin-branching papillae and exhibit high grade dysplasia. 